\title{
Investigating the effect of a platelet additive solution on apheresis platelet and fibrin network ultrastructure
}

\author{
E. Pretorius ${ }^{\mathrm{a}, *}$, R. Crookes ${ }^{\mathrm{b}}$, H.M. Oberholzer ${ }^{\mathrm{a}}$, W.J. van der Spuy ${ }^{\mathrm{a}}$ \\ ${ }^{a}$ Department of Anatomy, University of Pretoria, South Africa \\ ${ }^{\mathrm{b}}$ South African National Blood Service, South Africa
}

\section{A R T I C L E I N F O}

\section{Article history:}

Available online $\mathrm{xxxx}$

\section{Keywords:}

Apheresis

First-generation platelet additive solution

Ultrastructure

Fibrin networks

Platelets

\begin{abstract}
A B S T R A C T
In thrombotic events and diseases such as cancer, HIV/AIDS, dysfibrinogenaemia, as well as acute incidents (e.g. burn wounds), ultrastructure of platelets and fibrin networks change. In the current study, we compare the ultrastructure of platelets and fibrin networks of apheresis platelets stored in citrated human plasma (CP) and in a first-generation platelet additive solution (PAS) (T-Sol), to that of fresh donor plasma (FP). Eighteen apheresis platelet donors donated platelets on Trima ${ }^{\circledR}$-Accel ${ }^{\mathrm{TM}}$ V5.2 and V5.1 cell separators. Six collections were stored for five days in autologous citrated plasma (CP); six collections were stored in $40 \%$ citrated human plasma and $60 \%$ PAS solution (CP/PAS) controlled, for the duration of storage, at a constant temperature $\left(22 \pm 2^{\circ} \mathrm{C}\right.$ ) with continuous flat-bed agitation; and six collections were stored in conditions uncontrolled for temperature and without continuous agitation. On days 1, 3 and 5, equal volumes of human thrombin were mixed with platelets collected in either $\mathrm{CP}$ or $\mathrm{CP} / \mathrm{PAS}$ to form a coagulum (fibrin network containing platelet aggregates), followed by preparation for scanning electron microscopy. Results were compared with platelets and fibrin networks in FP. Typically, in FP, platelet aggregates with smooth membranes and pseudopodia are seen and fibrin networks arrange to form major, thick fibers and scattered, minor, thin fibers. On day 1 , in CP and in all CP/PAS units, platelet ultrastructure compared well to that of FP, although the fibrin fibers were denser, with the minor fibers forming a matted layer over the major fibers. On day 3, in platelet units uncontrolled for temperature and without continuous agitation during storage, some platelet aggregates in CP/PAS showed typical apoptotic morphology, with shrinkage and membrane damage, but comparable fibrin networks were present. On day 5 however, in those units where storage conditions were uncontrolled and where the $\mathrm{pH}$ had decreased to below 6.4, no platelet aggregates were seen and fibrin was arranged into short, lumpy masses with no separate major or minor fibrin fibers visible. In those units stored at $22{ }^{\circ} \mathrm{C}$ with continuous flat-bed agitation, where $\mathrm{pH}$ was maintained $>7.0$, ultrastructure of platelets and fibrin network in $\mathrm{CP} / \mathrm{PAS}$ was typical and similar to $\mathrm{FP}$ and $\mathrm{CP}$ at the end of five days of storage. Examining platelet and fibrin network ultrastructure may be useful, in addition to conventional laboratory analysis, in assessing the viability and potential clinical efficacy of platelets for transfusion and could play a role in the evaluation of new generation platelet additive solutions.
\end{abstract}

(c) 2010 Elsevier Ltd. All rights reserved.

\footnotetext{
* Corresponding author. Address: BMW Building, P.O. Box 2034, Faculty of Health Sciences, University of Pretoria, Pretoria 0001, South Africa. Tel.: +27 12319 2533; fax: +27 123192240 .

E-mail address: resia.pretorius@up.ac.za (E. Pretorius).
}

\section{Introduction}

Platelet transfusions have become integral to the medical management of a wide range of clinical conditions. The storage period of platelets collected from blood donors 
is limited because of the adverse changes in platelet morphology and function that take place during storage, reducing their clinical efficacy. Various strategies have been devised to minimize the storage lesion and lengthen the duration of storage, including suspending donated platelets in a synthetic medium. The development of platelet additive solutions (PAS) has several potential advantages. It may reduce the incidence of allergic transfusion reactions, facilitate $\mathrm{ABO}$-incompatible platelet transfusions, enable pathogen inactivation, and make plasma available for other purposes (e.g. for fractionation into blood derivatives) [1]. It is however important to ensure that platelets stored in a platelet additive solution maintain adequate function. Parameters used to measure the viability of donated platelets include the swirl phenomenon, $\mathrm{pH}$, p-selectin and, in vivo, post transfusion platelet increment and corrected count increment (CCI). Several compounds in platelet additive solutions such as citrate, acetate, phosphate, potassium, magnesium and glucose, have all been shown to be important $[2,3]$.

Although ample information regarding the in vitro and in vivo impact of PAS on platelet viability is available, no ultrastructural studies have been performed on platelet and fibrin networks in the presence of PAS. The current study therefore investigates the effect of the platelet additive solution, T-Sol, on apheresis platelet and fibrin network ultrastructure.

\section{Materials and methods}

\subsection{Platelet collection: controls}

Fresh platelet rich plasma (FP) was prepared by drawing $40 \mathrm{ml}$ of blood from six healthy individuals (Research Ethical Committee of the University of Pretoria, South Africa; ethical clearance number 151/2006).

\subsection{Apheresis platelet collection}

Eighteen apheresis platelet donors donated platelets on the Trima ${ }^{\circledR}$-Accel ${ }^{\mathrm{TM}}$ V5.2 and V5.1 cell separators. The following procedures were followed:

- Six collections were stored for five days in autologous citrated plasma (CP); the units were tested on days 1 , 3 and 5 of storage for platelet swirl, pH, p-selectin and by scanning electron microscopy (SEM).

- Six collections (referred to as uncontrolled CP/PAS) were stored in conditions uncontrolled for temperature and without continuous agitation. The units were tested on days 1, 3 and 5 of storage for platelet swirl, $\mathrm{pH}$, p-selectin and by scanning electron microscopy (SEM).

- Six collections (referred to as controlled CP/PAS) were stored in $40 \%$ citrated human plasma and $60 \%$ PAS solution (CP/PAS) controlled, for the duration of storage, at a constant temperature $\left(22 \pm 2{ }^{\circ} \mathrm{C}\right)$ and with continuous flat-bed agitation. On day 5 these collections were studied using the SEM.
In all $\mathrm{CP} / \mathrm{PAS}$ units, $\mathrm{T}$-Sol was added at the end of the apheresis procedure and each collection was split into two therapeutic doses (containing a minimum of $3 \times 10^{11}$ platelets). All units were irradiated with gamma-irradiation (30 Gy) on day 1 of storage.

\subsection{Formation of clot for scanning electron microscopy (SEM)}

On days 1, 3 and 5, equal volumes of human thrombin were mixed with either $\mathrm{FP}, \mathrm{CP}$ or $\mathrm{CP} / \mathrm{PAS}$ to form a coagulum (fibrin network containing platelet aggregates). Human thrombin (provided by the South African National Blood Service) was used to prepare all fibrin clots. The thrombin solution is at a concentration of $20 \mathrm{U} / \mathrm{ml}$ and is made up in a biological buffer containing $0.2 \%$ human serum albumin.

When thrombin is added, fibrinogen is converted to fibrin and intracellular platelet components, e.g. transforming growth factor, platelet-derived growth factor and fibroblastic growth factor are released into the coagulum. The coagulum was formed on a $0.2 \mu \mathrm{m}$ Millipore membrane and this Millipore membrane was then placed in a Petri dish on filter paper dampened with phosphate buffered saline (PBS) to create a humid environment and placed at $37^{\circ} \mathrm{C}$ for $10 \mathrm{~min}$. This was followed by a washing process where the Millipore membranes with the coagula were placed in PBS and magnetically stirred for varying times (45, 90 and $120 \mathrm{~min}$ ). This was done to remove any blood proteins trapped within the fibrin network $[4,5]$.

\subsection{Preparation of washed fibrin clot for SEM}

Washed fibrin clots were fixed in $2.5 \%$ glutaraldehyde in Dulbecco's phosphate buffered saline (DPBS) buffer with a $\mathrm{pH}$ of 7.4 for $1 \mathrm{~h}$. Each fibrin clot was rinsed three times in phosphate buffer for $5 \mathrm{~min}$ before being fixed for $1 \mathrm{~h}$ with $1 \%$ osmium tetraoxide $\left(\mathrm{OsO}_{4}\right)$. The samples were rinsed thrice with distilled water for 5 min and were dehydrated serially in $30 \%, 50 \%, 70 \%, 90 \%$ and three times with $100 \%$ ethanol. The SEM procedures were completed by drying of the material with hexamethyldisilazane (HMDS) [6], mounting, coating with ruthenium tetraoxide (SPI Supplies, West Chester USA) and examination of the tissue with a ZEISS ULTRA plus FEG scanning electron microscope with InLens capabilities.

\section{Results and discussion}

All $\mathrm{CP}$ and $\mathrm{CP} / \mathrm{PAS}$ units showed normal platelet morphology, swirl response, $\mathrm{pH}$ and p-selection expression on the day of collection. On days 3 and 5 of storage, platelets collected in citrated plasma (Collection 1 ) showed normal platelet morphology and in vitro test results were within standard quality parameters (11/12 units). Both Annexin V and CD62P showed increased expression over time, indicating that the number of platelets activated increased from day 1 to day 5 . Only 2 of 12 (split) units of platelet concentrate stored in $\mathrm{CP} / \mathrm{PAS}$ and uncontrolled 
for temperature and without continuous agitation (Collection 2), showed the swirl phenomenon on day 3 of storage. The $\mathrm{pH}$ of these CP/PAS units decreased significantly over time (Table 1).

Fig. 1a and b shows ultrastructure of a typical aggregated platelet mass and fibrin network prepared from citrated blood (FP). On aggregation, platelets form a cluster that possesses a smooth membrane with a few membrane pores visible. Also, slight bulging areas or pseudopodia are typically present, indicated by white arrows in Fig. 1a. Fibrin fibers of FP are seen in Fig. 1b. Typically, thick, major fibers and thin, minor fibers are seen in a coagulum, with the major fibers being the most prominent and the minor fibers weaved between the major fibers.

Ultrastructure results of $\mathrm{CP}$ and uncontrolled $\mathrm{CP} / \mathrm{PAS}$ units are shown in Fig. 2. Micrographs of days 1, 3 and 5 are shown for both $\mathrm{CP}$ and $\mathrm{CP} / \mathrm{PAS}$. Fig. 2a-c shows $\mathrm{CP}$ results. On day 1 , platelets appear similar to FP, with smooth membranes and pseudopodia present (Fig. 2a - platelet aggregate indicated by thin, white arrows). The CP fibrin network appears denser and more matted than that of FP (thick, white arrow). Platelets stored in CP appear similar on days 3 and 5 when compared to day 1 (Fig. 2b and c). Some areas in the coagulum tend to form a thinner distributed mat of thin, minor fibers, but both major and minor fibers are visible in all samples.

Table 1

$\mathrm{pH}$ of $\mathrm{CP}$ and all $\mathrm{CP} / \mathrm{PAS}$ units on day 5 .

\begin{tabular}{lc}
\hline Collection type & Day 5 \\
\hline $\mathrm{CP}$ & 7.2 \\
$\mathrm{CP}$ & 7.1 \\
$\mathrm{CP}$ & 7.3 \\
$\mathrm{CP}$ & 7.12 \\
$\mathrm{CP}$ & 7.28 \\
$\mathrm{CP}$ & 6.14 \\
Uncontrolled CP/PAS units & \\
$\mathrm{CP} / \mathrm{PAS}$ & 6.6 \\
$\mathrm{CP} / \mathrm{PAS}$ & 5.9 \\
$\mathrm{CP} / \mathrm{PAS}$ & 6.1 \\
$\mathrm{CP} / \mathrm{PAS}$ & 5.82 \\
$\mathrm{CP} / \mathrm{PAS}$ & 6.03 \\
$\mathrm{CP} / \mathrm{PAS}$ & 6.85 \\
Controlled CP/PAS units & \\
All six collections in CP/PAS & $>7.1$ \\
\hline
\end{tabular}

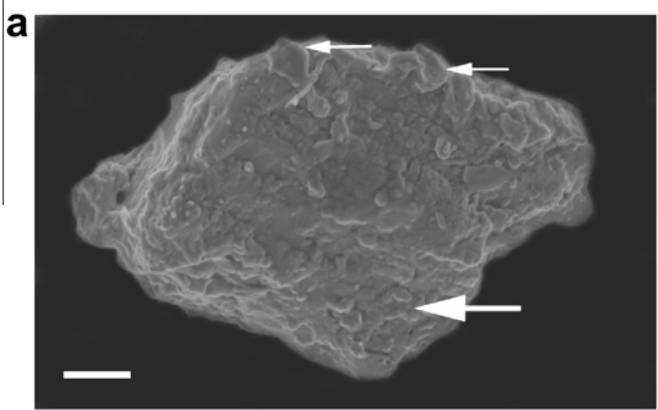

Fig. 2d-g shows fibrin ultrastructure of platelets stored in uncontrolled CP/PAS, for days 1, 3 and 5. On day 1 platelet aggregates are comparable to both FP and $\mathrm{CP}$ platelets (thin, white arrows); however, the fibrin seems more matted than present in the CP. On day 3, fibrin appears the same as on day 1 , however, two variants of platelet aggregates are seen in the coagulum (Fig. 2e and f). In the coagulum there are platelets that compare well to that of FP and CP (Fig. 2e), however, a number of platelets show an apoptotic appearance (Fig. 2f). Apoptotic platelets are characterized by a morphological pattern of cell death marked by cell shrinkage, condensation of chromatin, the formation of cytoplasmic blebs and fragmentation of the cell membrane into membrane-bound apoptotic bodies that are eliminated by phagocytosis. The occurrence of apoptosis or programmed cell death in patho-physiological settings can be the result of human disease [7]. Apoptosis is characterized by a dramatic loss in cell volume, membrane blebbing, rearrangement of the cytoskeleton and loss of contact with adjacent cells [7]. Cellular changes are partly caused by proteolytic clearance of intracellular components that regulate the integrity of the cytoskeleton [7]. Wyllie et al. [8] succinctly defined apoptosis as a form of cell death characterized by cell shrinkage and nuclear condensation. Apoptotic platelet aggregates have also previously been seen in patients with HIV/AIDS [5,9].

Fig. $2 \mathrm{~g}$ shows the ultrastructure of platelets from $\mathrm{CP} / \mathrm{PAS}$ units uncontrolled for temperature and without constant agitation during storage, where $\mathrm{pH}$ had decreased to below 6.4 , on day 5 . Here, no platelets were seen in any of the six donated units and no typical individual fibrin fibers were distinguished. Instead, fibrin was arranged in tight, short, globular masses. It is likely that this changed morphology is due to the decrease in $\mathrm{pH}$ that was observed in the uncontrolled CP/PAS units (Table 1).

The six controlled CP/PAS apheresis platelet units were stored at a constant temperature $\left(22 \pm 2^{\circ} \mathrm{C}\right)$ with continuous flat-bed agitation for the duration of storage. The $\mathrm{pH}$ remained $>7.0$ and swirling was observed to the end of day 5 in all units. Fig. 3a shows a platelet aggregate and Fig. 3b shows fibrin network ultrastructure on day 5. Platelet aggregates do not differ from control platelets collected in fresh plasma (day 1), and fibrin networks look very similar to those seen on day 5 - Fig. $2 c$.

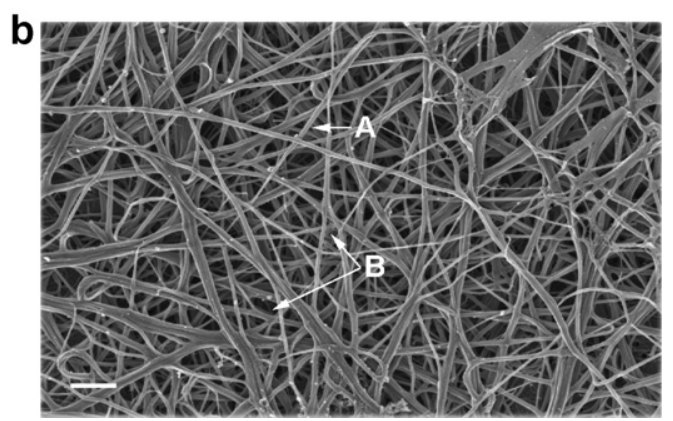

Fig. 1. (a) Micrograph showing platelet mass prepared from fresh platelet rich plasma (FP). Thick, white, arrow = platelet mass showing smooth membrane; thin, white arrow $=$ pseudopodia. Scale $=1 \mu \mathrm{m}$. (b) Micrograph showing fibrin network prepared from fresh platelet rich plasma (FP). Label A = major fibers; label $\mathrm{B}=$ minor fibers. Scale $=1 \mu \mathrm{m}$. 
a
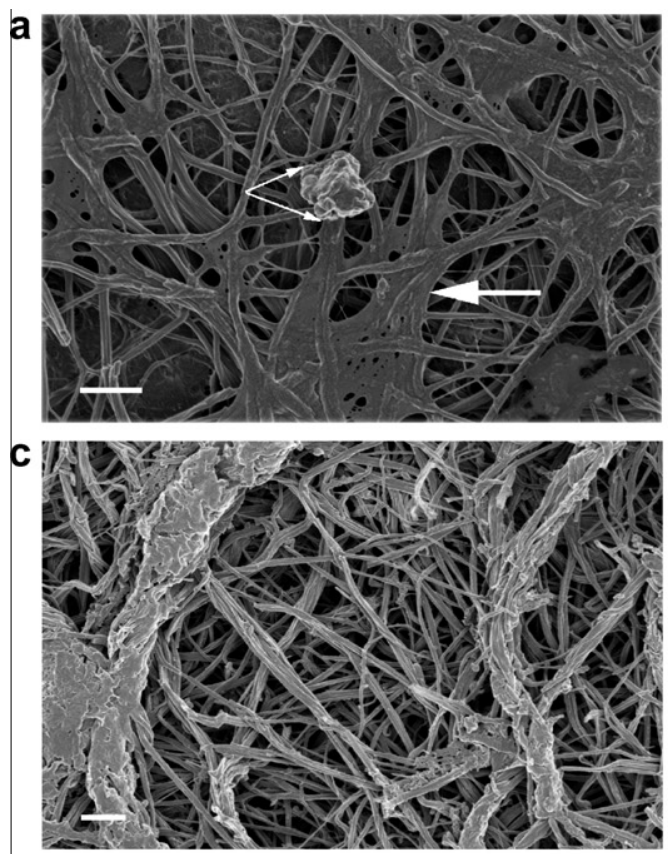

e

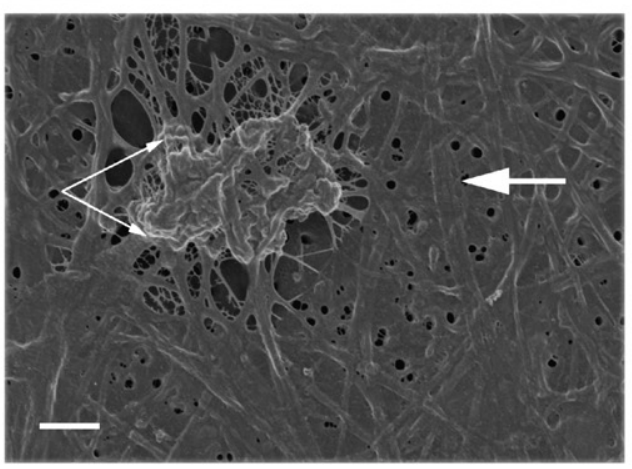

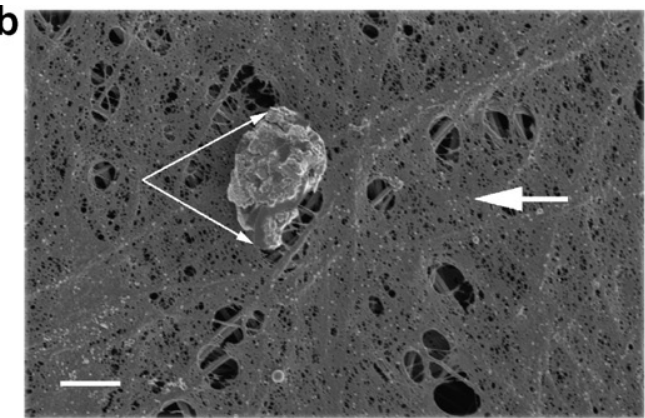

d

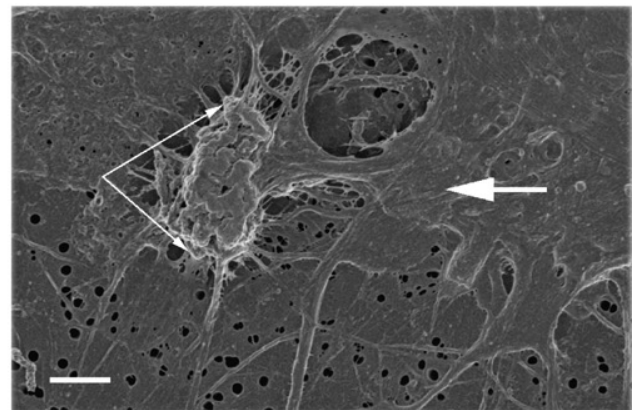

f

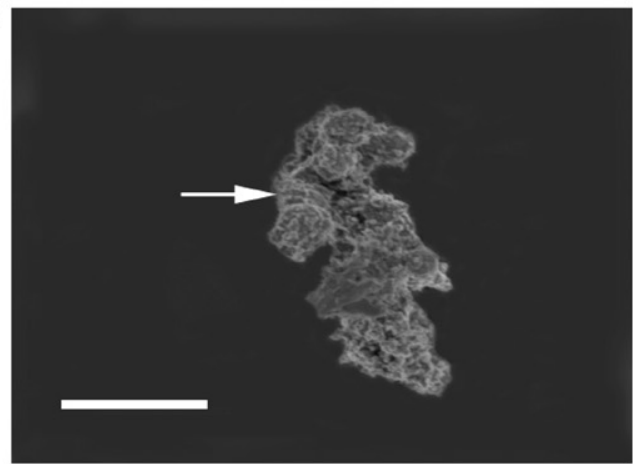

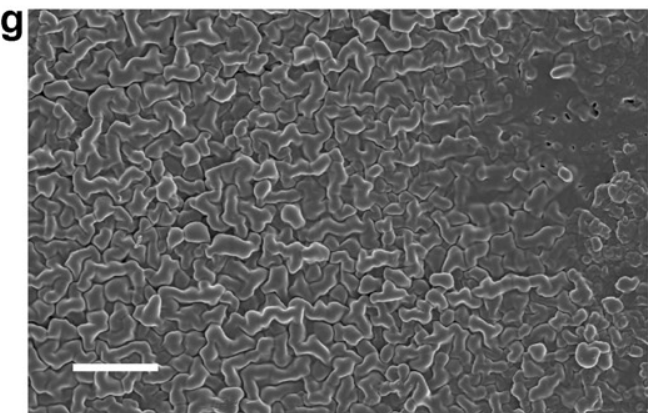

Fig. 2. Micrographs showing differences between platelet and fibrin network ultrastructure during storage. (a) Platelet rich plasma (CP) (day 1). Thick, white, arrow = matted fibrin; thin, white arrow = platelet mass. Scale $=1 \mu \mathrm{m}$. (b) Platelet rich plasma $(\mathrm{CP})($ day 3$)$. Thick, white, arrow $=$ matted fibrin; thin, white arrow $=$ platelet mass. Scale $=1 \mu \mathrm{m}$. (c) Platelet rich plasma (CP) (day 5). Individual fibrin fibers visible. Scale $=1 \mu \mathrm{m}$. $(\mathrm{d}) \mathrm{Uncontrolled} \mathrm{CP} / \mathrm{PAS}$ : platelet rich plasma with T-Sol (CP/PAS) (day 1) stored in conditions uncontrolled for temperature and without constant agitation. Thick, white, arrow $=$ matted fibrin; thin, white arrow $=$ platelet mass. Scale $=1 \mu \mathrm{m}$. (e) Uncontrolled $\mathrm{CP} / \mathrm{PAS}$ : platelet rich plasma with T-Sol $(\mathrm{CP} / \mathrm{PAS})$ with normal appearing platelet mass (day 3 ). Thick, white, arrow = matted fibrin; thin, white arrow = platelet mass. Scale $=1 \mu \mathrm{m}$. (f) Uncontrolled $\mathrm{CP} / \mathrm{PAS}$ : platelet rich plasma with T-Sol (CP/PAS) showing apoptotic platelet mass (day 3 ). Thin, white arrow = membrane of platelet mass atypical. Scale $=1 \mu \mathrm{m}$. ( $\mathrm{g}$ ) Uncontrolled CP/PAS: platelet rich plasma with T-Sol (CP/PAS) (day 5). Atypical fibrin network. No platelet masses visible. Scale $=1 \mu \mathrm{m}$.

Apheresis platelets collected and stored in citrated plasma $(\mathrm{CP})$ and in $\mathrm{CP} / \mathrm{PAS}$, controlled for temperature and with constant agitation during storage, fulfilled standard quality criteria through day 5 of storage. However, platelets collected in T-Sol (CP/PAS) and stored in conditions not controlled for temperature, and without continuous agitation, were not suitable for transfusion after day 3 of storage. Ultrastructure correlated with general quality control 

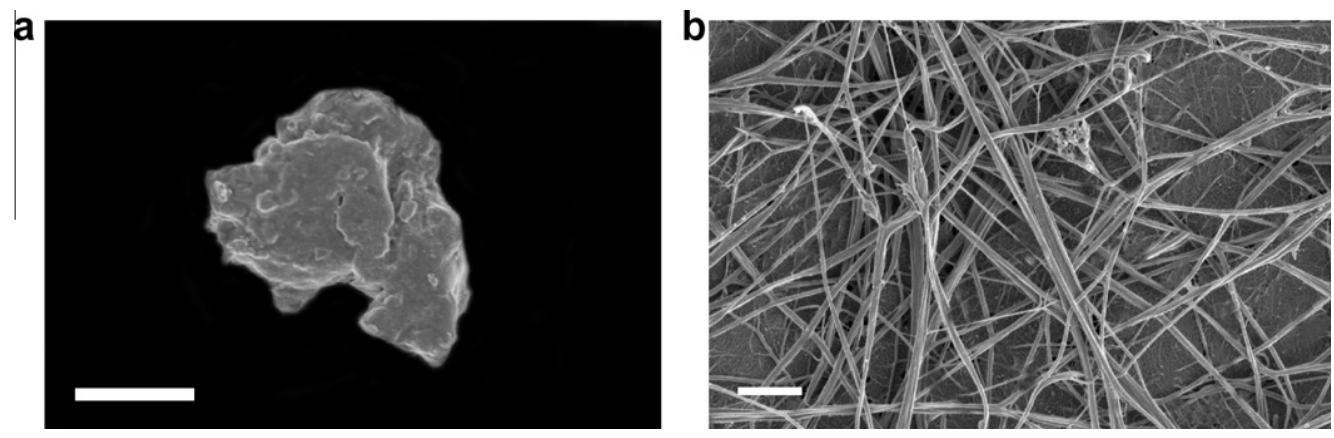

Fig. 3. (a) Controlled $\mathrm{CP} / \mathrm{PAS}$ : platelet morphology from platelet rich plasma $(\mathrm{CP})$ (day 5), stored in conditions controlled for temperature $\left(22{ }^{\circ} \mathrm{C}\right)$ and with constant flat-bed agitation; $\mathrm{pH}>7.1$; swirling present. Scale $=1 \mu \mathrm{m}$. (b) Controlled CP/PAS: fibrin network morphology from platelet rich plasma (CP) (day 5) stored in conditions controlled for temperature $\left(22^{\circ} \mathrm{C}\right)$ and with constant flat-bed agitation; $\mathrm{pH}>7.1$; swirling present. Scale $=1 \mu \mathrm{m}$.

laboratory results, including $\mathrm{pH}$ and swirl. Uncontrolled $\mathrm{CP} / \mathrm{PAS}$ units, showed some apoptotic platelet formation on day 3 and, on day 5, no platelets were seen in the coagulum. Fibrin networks were coagulated into an unrecognizable mat of short, globular areas. This was not found in CP units or in controlled CP/PAS units. We conclude that a lowered $\mathrm{pH}$ causes the fibrin network changes.

Examining platelet and fibrin network ultrastructure provides an additional means of assessing the viability and potential clinical efficacy of platelets for transfusion and could play a role in the evaluation of new generation platelet additive solutions.

\section{Acknowledgement}

The authors thank Sr. Winnie Poongavanum, Sr. Carolina Strydom and staff of the South Afrian National Blood Service's Pretoria Apheresis Unit, as well as SANBS staff in the Blood Proceesing and Quality Departments, for their expert assistance.

\section{References}

[1] Ringwald J, Zimmermann R, Eckstein R. The new generation of platelet additive solution for storage at 22 degrees C: development and current experience. Transfus Med Rev 2006;20(2):158-64.
[2] Ringwald J, Haager B, Krex D, Zimmermann R, Strasser E, Antoon M, et al. Impact of different hold time before addition of platelet additive solution on the in vitro quality of apheresis platelets. Transfusion 2006;46(6):942-8.

[3] Gulliksson H, AuBuchon JP, Cardigan R, et al. Storage of platelets in additive solutions: a multicentric study of the in vitro effects of potassium and magnesium. Vox Sang 2003;85:199-205.

[4] Pretorius E, Briedenhann S, Marx J, Smit E, van der Merwe CF, Pieters $\mathrm{M}$, et al. Ultra-structural comparison of the morphology of three different platelet and fibrin fiber preparations. Anat Rec 2007;290:188-98.

[5] Pretorius E, Oberholzer HM, Smit E, Steyn E, Briedenhann S, Franz RC. Ultrastructural changes in platelet aggregates of HIV patients: a scanning electron microscopy study. Ultrastruct Pathol 2008;32:75-9.

[6] Araujo JC, Téran FC, Oliveira RA, Nour EAA, Montenegro MAP, Campos $J R$, et al. Comparison of hexamethyldisilazane and critical point drying treatments for SEM analysis of anaerobic biofilms and granular sludge. J Electron Microsc 2003;52(4):429-33.

[7] Gottlieb RA. Apoptosis. In: Lichtman MA, Kipps J, Kaushansky K, Beutler E, Seligsohn U, Prehal JT, editors. Williams' haemotology. New York: McGraw Hill; 2006.

[8] Wyllie AH, Kerr JF, Currie AR. Cell death: the significance of apoptosis. Int Rev Cytol 1980;68:251-306.

[9] Pretorius E, Oberholzer HM, Smit E, Steyn E, Briedenhann S, Franz RC. Investigating the ultrastructure of platelets of HIV patients treated with the immuno-regulator, Canova: a qualitative scanning electron microscopy study. Histol Histopathol 2009;24:399-405. 